DOE/BC/14953-23

(OSTI ID: 14229)

INCREASÉD OIL PRODUCTION AND RESERVES FROM IMPROVED COMPLETION TECHNIQUES IN THE BLUEBELL FIELD, UINTA BASIN, UTAH

Quarterly Technical Progress Report

January 1, 1998-March 31, 1998

By

Craig D. Morgan

Milind D. Deo

Report Issue Date: March 1998

Performed Under Contract No. DE-FC22-92BC14953

Utah Geological Survey

Salt Lake City, Utah 


\section{DISCLAIMER}

This report was prepared as an account of work sponsored by an agency of the United States Government. Neither the United States Government nor any agency thereof, nor any of their employees, makes any warranty, expressed or implied, or assumes any legal liability or responsibility for the accuracy, completeness, or usefulness of any information, apparatus, product, or process disclosed, or represents that its use would not infringe privately owned rights. Reference herein to any specific commercial product, process, or service by trade name, trademark, manufacturer, or otherwise does not necessarily constitute or imply its endorsement, recommendation, or favoring by the United States Government or any agency thereof. The views and opinions of authors expressed herein do not necessarily state or reflect those of the United States Government.

This report has been reproduced directly from the best available copy. 


\section{DISCLAIMER}

Portions of this document may be illegible in electronic image products. Images are produced from the best available original document. 
$\mathrm{DOE} / \mathrm{BC} / 14953-23$

Distribution Category UC-122

Increased Oil Production and Reserves from Improved Completion Techniques in the Bluebell Field, Uinta Basin, Utah

\author{
By \\ Craig D. Morgan \\ Milind D. Deo
}

November 1999

Work Performed Under Contract No. DE-FC22-92BC14953

\author{
Prepared for \\ U.S. Department of Energy \\ Assistant Secretary for Fossil Energy
}

Gary Walker, Project Manager

National Petroleum Technology Office

P.O. Box 3628

Tulsa, OK 74101

Prepared by

Utah Geological Survey

1594 West North Temple, Suite 3110

Salt Lake City, UT 84114 
INCREASED OIL PRODUCTION AND RESERVES FROM

IMPROVED COMPLETION TECHNIQUES IN THE

BLUEBELL FIELD, UINTA BASIN UTAH

Quarterly Technical Report

Reporting Period: Start Date:01/01/1998End Date: 03/31/1998

Authors:

CRAIG D. MORGAN

MILIND D. DEO

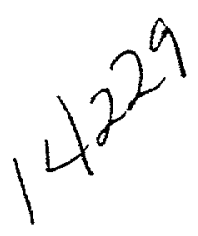

Report Issue Date: 04/04/1998

DE-FC22-92BC14953 -23

CRAIG D. MORGAN

Utah Geological Survey

Box 146100

Salt Lake City, UT 84114

Milind D. Deo

Dept. of Fuels \& Chemical Eng

University of Utah

3297 Merrill Eng Bldg

SLC UT 84112 


\author{
Quarterly Technical Progress Report \\ 01/01/98 - 03/31/98 \\ $18^{\text {th }}$ Quarter of the Project
}

\title{
Increased Oil Production And Reserves From Improved Completion Techniques In The Bluebell Field, Uinta Basin, Utah
}

Contract DE-FC22-92BC14953

\author{
Gary Walker \\ U.S. Department of Energy \\ National Petroleum Technology Office \\ Contracting Officer's Representative
}

\author{
Craig D. Morgan \\ Program Manager \\ Utah Geological Survey \\ (801) $537-3370$
}

US/DOE Patent Clearance is not required prior to the publication of this document 


\section{Objectives}

The objective of this project is to increase oil production and reserves in the Uinta Basin by demonstrating improved completion techniques. Low productivity of Uinta Basin wells is caused by gross production intervals of several thousand feet that contain perforated thief zones, water-bearing zones, and non-perforated oil-bearing intervals. Geologic and engineering characterization and computer simulation of the Green River and Wasatch Formations in the Bluebell field will determine reservoir heterogeneities related to fractures and depositional trends. This will be followed by drilling and recompletion of several wells to demonstrate improved completion techniques based on the reservoir characterization. Transfer of the project results will be an ongoing component of the project.

\section{SUMMARY OF TECHNICAL PROGRESS}

\section{Development of a Parallel Processing Fractured Reservoir Simulator}

A portable, parallel, fractured-reservoir simulator was developed. The development and performance of the simulator on a shared-memory machine (Silicon Graphics Power Challenge) were reported in earlier quarterly and annual reports.

The performance of the parallel program was also studied on a distributed memory machine, the IBM SP which has 64 nodes of which 8 nodes are 66 megahertz (MHZ) processors with 128 megabytes (Mbytes) of local memory and the remaining 56 nodes were $120 \mathrm{MHZ}$ processors. The local memory configuration for the remaining 56 nodes was as follows: 46 nodes with 128 Mbytes, four with 256 Mbytes, two with 512 Mbytes, and four with 1 gigabyte (Gbyte). Each node had 2.2 Gbytes of local hard disk space. The communications between different nodes are performed through an external ethernet connection and a high performance switch that allows communications between any two nodes. The performance of the parallel program was studied with the 4, $120 \mathrm{MHZ}$ processor nodes with 1 Gbyte of local memory. The performance was studied for the same four data sets that were examined on the shared memory machine. These had grid configurations of $16 \times 16 \times 16,32 \times 32 \times 16,64 \times 64 \times 16$, and $128 \times 128 \times 16$ respectively. The times required for various computations are compared for one, two, and four processor configurations.

The time required to calculate the coefficient matrices are compared in Fig. 1. For the smallest data set (16X16X16) the time required does not decrease significantly as the number of processors are increased. For the $32 \times 32 \times 16$ data set, as the number of processors is increased from one to two and four, the time required decreases though the decrease is not very significant. For the larger data sets, $64 \times 64 \times 16$ and $128 \times 128 \times 16$, the time required decreases significantly as the number of processors is increased from one to four (by a factor of 2.5 for the four processor configuration).

The times required to solve the fracture and matrix pressure equations are compared in Figs. 2 and 3. As can be seen from both the figures, there is no effect on the time required to solve the two equations as the number of processors is increased for the two small data sets, $16 \times 16 \times 16$ and $32 \times 32 \times 16$. For the $64 \times 64 \times 16$ and $128 \times 128 \times 16$ data sets, the time required 
increases as the number of processors is increased. The increase is very significant for solution of the matrix pressure equation. The total times for completion of one time step are compared in Fig. 4. Except for the largest data set (128X128X16), the time required to complete a single time step does not vary significantly with additional processors. For the largest data set, the time decreases as the number of processors is increased from one to two but increases as additional processors are used.

The results for the distributed memory machine are different than the shared memory machine. The message passing protocols used by the two machines are different. On the Power Challenge, each time a processor has to send a message it does so without waiting for a ready message from the processor that is supposed to receive the message. The processor performs the send and carries on to the next instruction. Conversely, on IBM SP, each processor waits for the ready signal from the receiving processor before it sends the data. This waiting takes place only for the cases where the size of the data to be communicated is at least four kilobytes (Kbytes). For the largest model (128X128X16) the size of the data to be communicated between the processors is greater than four Kbytes. The number of communications increases when the tridiagonal system of equations is solved with iterative method. As the number of processors is increased the time lost in waiting also goes up considerably. As can be seen from Figs. 2 and 3, the increase in time required to solve the system of equations is greater for the matrix equations compared to the fracture equations. This is because of the order in which the two equations are solved. The matrix equation is solved first, with initial guesses for fracture and matrix pressures from the previous time step. The results are then used to solve the fracture equations. This updated solution is used in solving the fracture equations. Thus, more iterations are required to solve the matrix equation requiring additional computing time.

The results of parallel computing on the distributed memory machine were rather disappointing. If the code is to be ported to a cluster of workstations, the cluster is expected to perform as a distributed memory virtual machine. The way in which the equations are solved and the communication protocol will have to be optimized to improve the performance of the code on distributed memory platforms; that work is underway. 


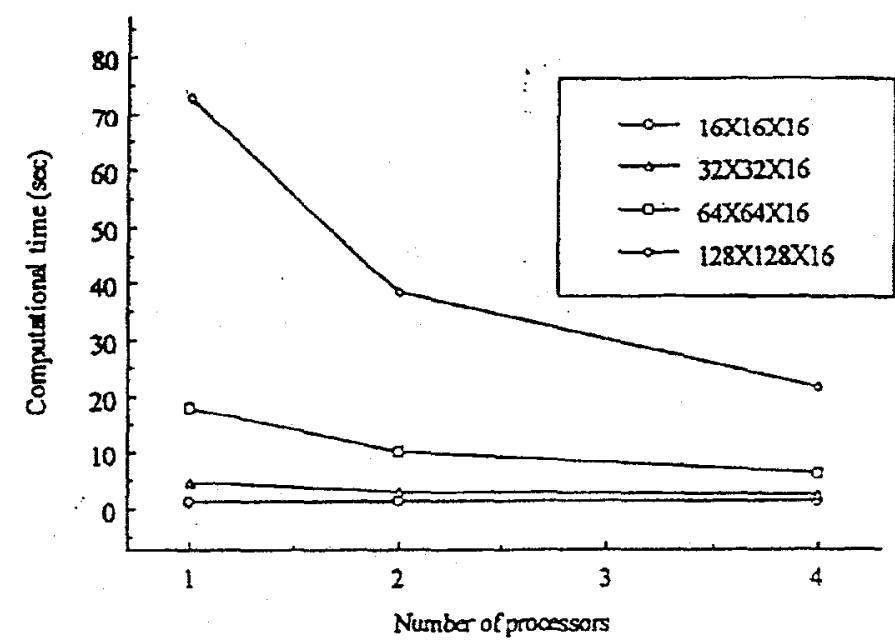

Figure 1. Comparison of computational times for calculation of coefficient matrices for the serial program (1 processor) and parallel program (2 and 4 processors) on IBM SP, a distributed memory machine (the four model grid configurations are shown in the explanation).

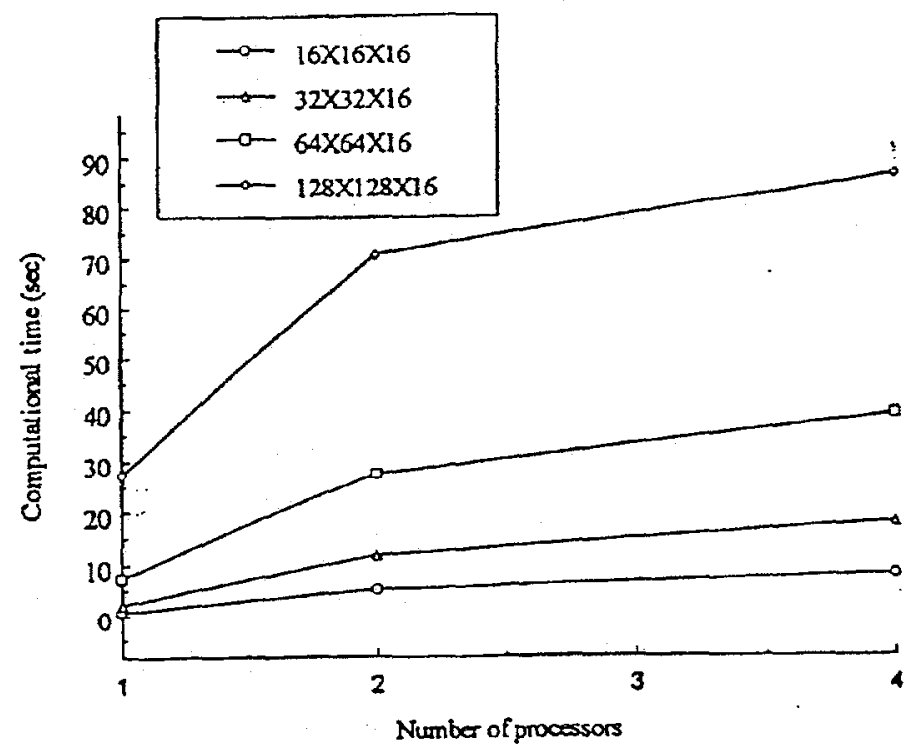

Figure 2. Comparison of computational times for the solution of fracture pressure equation on IBM SP, a distributed memory machine (the four model grid configurations are shown in the explanation). 


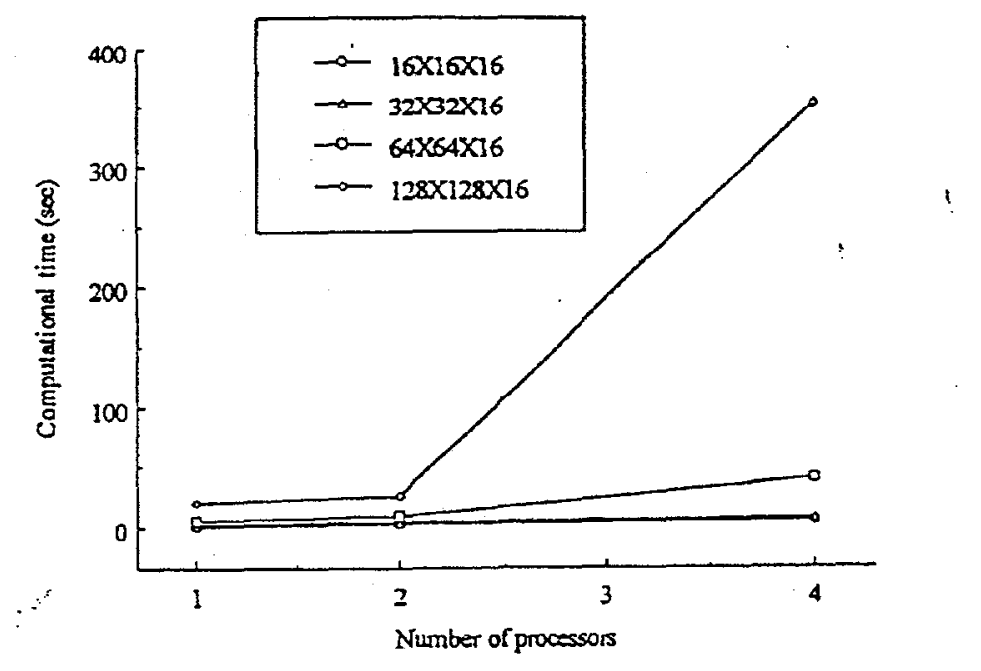

Figure 3. Comparison of computational times for the solution of matrix pressure equation on IBM SP, a distributed memory machine (the four model grid configurations are shown in the explanation).

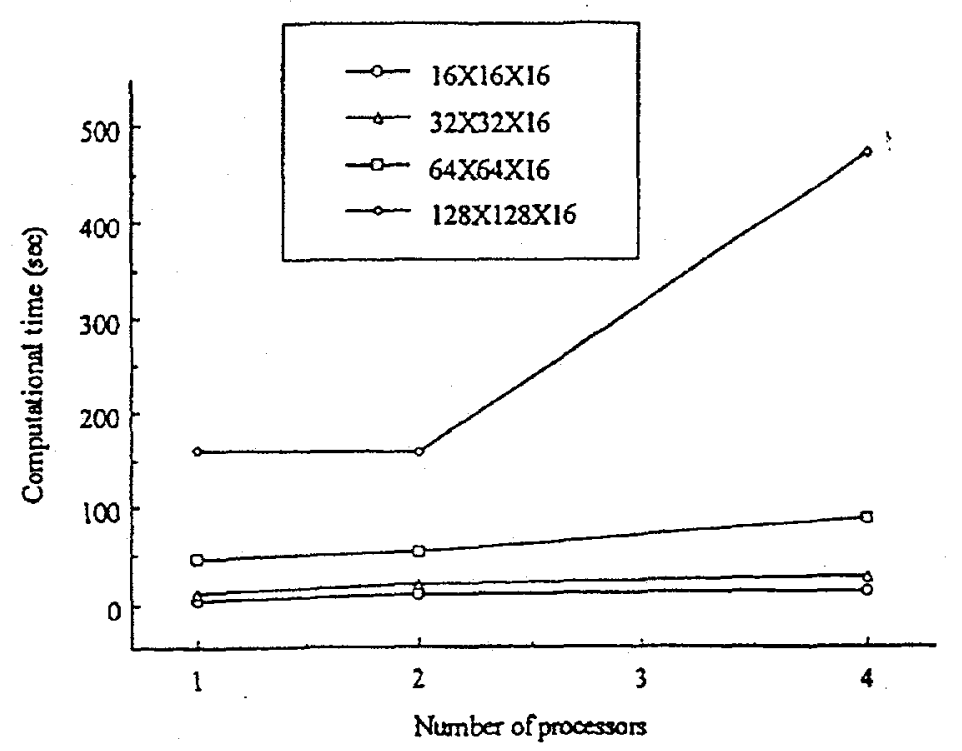

Figure 4. Comparison of total computational times for one time step on IBM SP, a distributed memory machine (the four model grid configurations are shown in the explanation). 


\section{Logging and Completing of a New Well}

The third step in the three-part demonstration is the logging and completion of a new well in the Bluebell field. The logging techniques used in the recompletion of the Michelle Ute 7-1 and Malnar Pike 17-1A1E wells will be used in the new well to help select fewer beds than are traditionally perforated. A staged-completion technique will be used rather than treating the entire gross perforated interval from one packer seat, which should provide a more effective treatment of individual beds.

Quinex Energy has begun drilling the Chasel 3-6A2 well (section 6, T. 1 S., R. 2W., UBM) which is scheduled to be the third demonstration well. The well will be drilled to a total depth of $16,000 \mathrm{ft}(4,876.8 \mathrm{~m})$ in the Tertiary Flagstaff Member of the Green River Formation (lower Wasatch transition in operator terminology). Neighboring wells have produced as little as $2,000 \mathrm{bbl}$ to over a million bbl of oil (Fig. 5). The location is in an area of regional northwest dip (Fig. 6). Hopefully, the well will be completed by July 1998. 


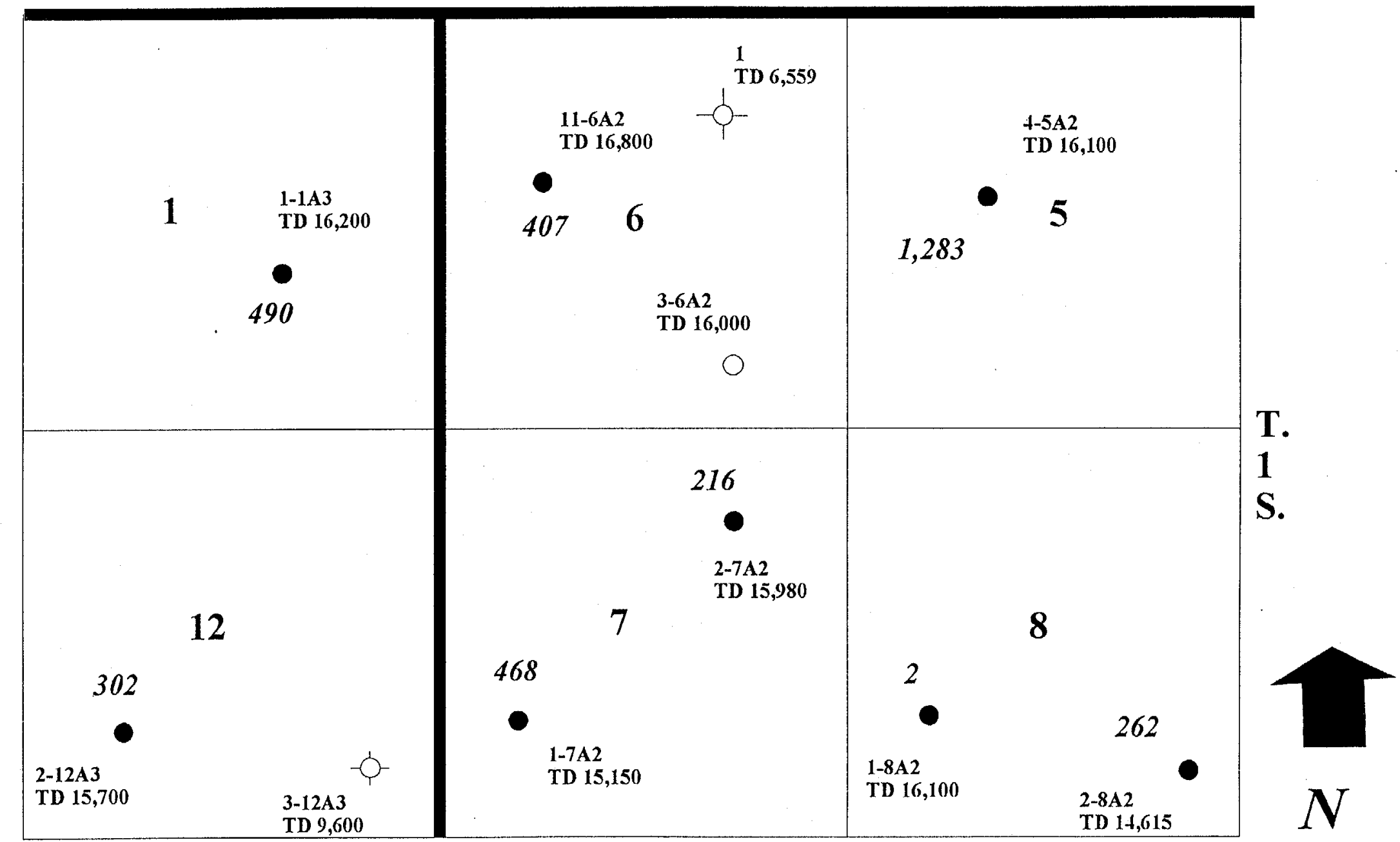

R. 3 W.

R. 2 W.

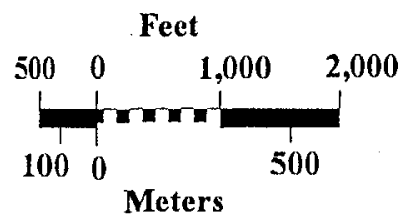

Figure 5. Cumulative production in thousands of barrels of oil, as of June 30, 1997. Data source: Utah Division of Oil, Gas and Mining. 


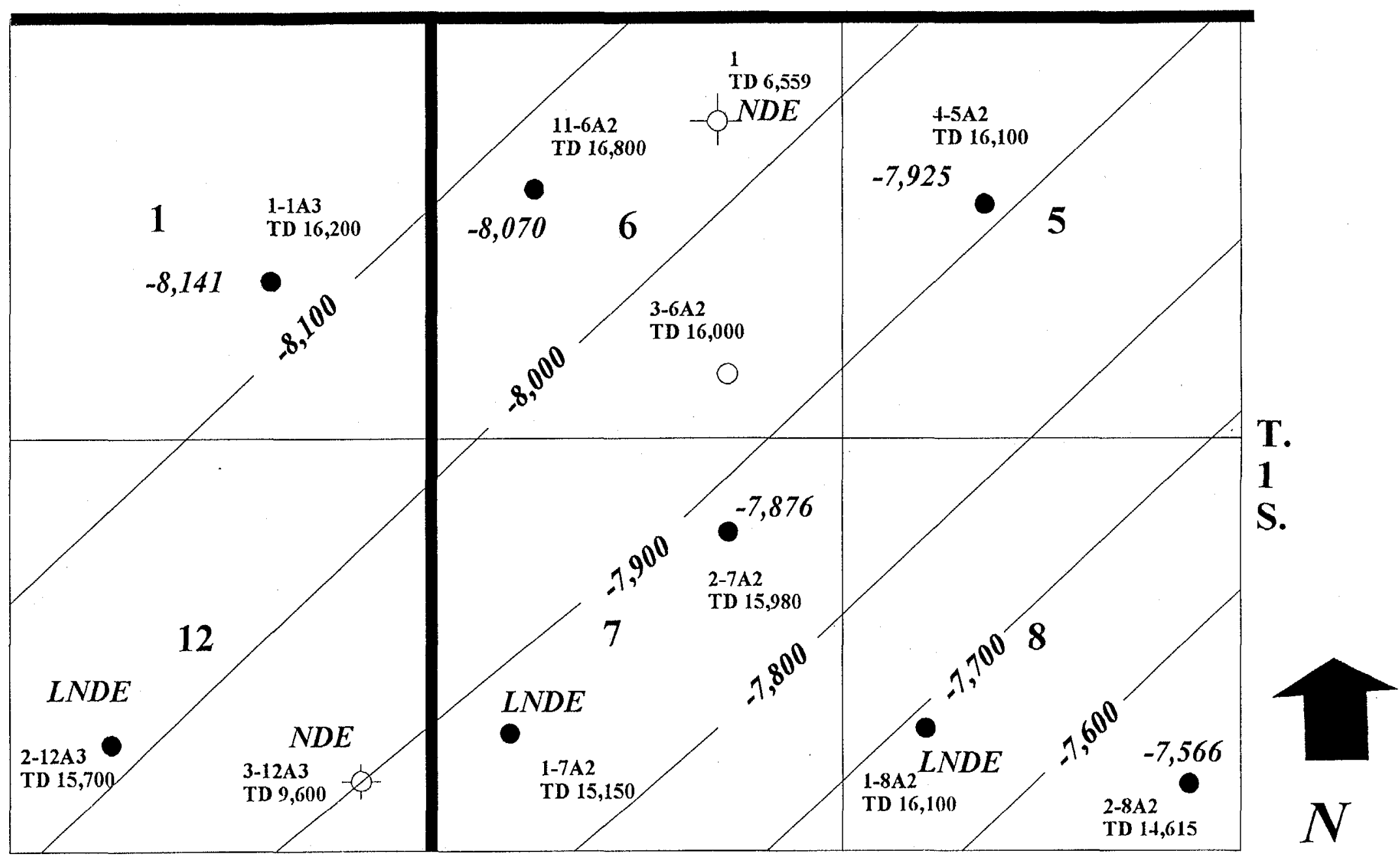

R. 3 W.

R. 2 W.

Feet

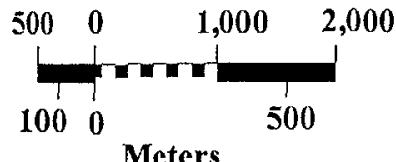

Figure 6. Structure contour top of the Flagstaff Member of the Green River Formation. Sea level datum, contour interval 100 feet. NDE- well is not deep enough, LNDE- logs are not deep enough. 


\section{Technology Transfer}

A presentation highlighting the cased-hole logging techniques used in the first two demonstrations was made at the DOE/PTTC sponsored workshop in Denver, CO., in January 1998. A poster session highlighting the results of the second demonstration will be presented at the AAPG Annual Convention in Salt Lake City, Utah, in May 1998.

The Utah Geological Survey maintains a Bluebell home page on its web site containing the following information: (1) a description of the project, (2) a list of project participants, (3) each of the Quarterly Technical Progress Reports, (4) a description of planned field demonstration work, (5) portions of the First and Second Annual Technical Reports with information on where to obtain complete reports, (6) a reference list of all publications that are a direct result of the project, (7) an extensive selected reference list for the Uinta Basin and lacustrine deposits worldwide, (8) daily activity reports of the Michelle Ute 7-1 and Malnar Pike 17-1 demonstration wells and, (9) abstracts prepared for the DOE/PTTC workshop on advanced logging techniques in Denver, CO., and the AAPG Annual Convention in Salt Lake City, UT . The home page address is http://www.ugs.state.ut.us/bluebell.htm 